\title{
ORGANISATION
}

\section{Freiwilliges Engagement wecken und fördern}

\author{
Rainer Hub
}

Freiwilliges Engagement ist gesamtgesellschaftlich eine Ressource der sozialen Entwicklung und der Weiterentwicklung des Sozialstaates mit seinen zivilgesellschaftlichen Akteuren. Es lebt alternativlos von der Freiwilligkeit Einzelner sich zu engagieren, die zum Gelingen der Agenda »Mehr freiwilliges Engagement wagen « einen wesentlichen Beitrag leisten.

23,4 Millionen Menschen engagieren sich in Deutschland freiwillig. Eine in den letzten Jahren um zwei Prozent leicht gestiegene Zahl. Das freiwillige Engagement im Sozialbereich als Akteur im Dritten Sektor ist signifikant am deutlichsten angewachsen. (1)

Die integrierende Funktion freiwilligen Engagements bei der Weiterentwicklung zu einer Tätigkeitsgesellschaft unterliegt vielfältigen Herausforderungen, die es zu gestalten gilt. Eine Zivilgesellschaft wird der integrativen Funktion nur gerecht, wenn sich deren breites Spektrum gezielt entwickelt. Die Begriffe »Arbeit « und »Wohlstand « gilt es neu zu definieren. Die Transformation einer Arbeitsgesellschaft in eine Tätigkeitsgesellschaft stellt eine enorme Herausforderung dar. Erwerbs-, Familien- und Engagementtätigkeiten müssen verbessert und gleichberechtigt miteinander vereinbar sein. Damit zusammen hängt die Weiterentwicklung der »Wohlstandsgesellschaft «Wohlstand als eine Frage des persönlichen Wohlbefindens. Zunehmend wird dies mehr mit Lebensqualität als mit dem Erwerb von Geld und Gütern zu tun haben. (2)

\section{Neue Potenziale von Staat, Zivilgesellschaft und Menschen}

Die Finanzierung von Arbeit und Engagement ist und wird dafür bunter und vielfältiger. Zahlreiche Stiftungsgründungen wie die »Bundesstiftung Diakonie« oder die »Stiftung Zeit für Menschen« werden zur Sozialen Arbeit zunehmend einen nicht unerheblichen Beitrag leisten. Neue, durchaus kritisch zu diskutierende Finanzierungsströme von sozialen Leistungen müssen sorgfältig implementiert werden. Eine starke Zivilgesellschaft mit Bürgerinnen und Bürgern, die in Eigeninitiative Selbstverantwortung übernehmen, ersetzt dabei nicht einen starken Staat: Im Gegenteil, er ist ihr Bündnispartner. Ein (Rechts-) Staat, der das (All-) Gemeinwohl schütz und fördert, kann nicht ohne starken Sozialstaat existieren. (3) Freiwilliges Engagement ist dabei eben nicht »irgendeine«

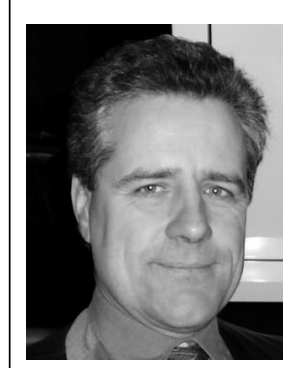

Rainer Hub (48) absolvierte ein sozial- und diakoniewissenschaftliches Studium an der Universität Heidelberg. Seit über zwölf Jahren ist er Referent für freiwilliges Engagement und Zivildienst beim Diakonischen Werk der Evangelischen Kirche in Deutschland e. $V$. E-Mailhub@diakonie.de

erbrachte Dienstleistung - nur unbezahlt. Setzt man auf diesen Ansatz, wird er sich als Irrweg erweisen.

Traditionell war die Förderung freiwilliges Engagement einzelner Menschen die wichtigste Aufgabe. Zunehmend muss es noch stärker die Aufgabe werden, engagementbegünstigende Rahmenbedingungen zu schaffen.

Für eine zukunftsfähige (Zivil-) Gesellschaft braucht es neben den sich freiwillig engagierenden Menschen (noch) mehr Kooperation und Vernetzung. Aufgaben, wie Soziales und Gesundheit, stellen dabei keine Ware dar und können weder an den Markt noch an die Zivilgesellschaft delegiert werden. Freiwilliges Engagement ist als ein unverzichtbarer Bestandteil eines Organisations- und Qualitätsentwicklungsprozesses zu verstehen. Darin sollte jeder Mensch die Möglichkeit haben, sich entsprechend seinen Wünschen, Fähigkeiten und individuellen Voraussetzungen engagieren zu können; je nach Lebenslage und Lebensphase. Bei jungen Menschen beispielsweise spielen Aspekte des Sammelns von Erfahrungen in Feldern Sozialer Arbeit und die Persönlichkeitsbildung eine Rolle. Vielen geht es in der Schulzeit darum, mit einem zeitlich befristeten Einsatz in der Sozialen Arbeit auszuloten, ob dies ein mögliches Berufsfeld für sie sein kann. Während Schülerinnen und Schüler oder Studierende sich eher zeitlich begrenzt mit wenigen Stunden pro Woche oder in den Ferien einbringen können, sind für Jugendliche nach der Schule vor allem Vollzeitdienste wie im Freiwilligen Sozialen Jahr attraktiv.

Bei diesen Überlegungen ist zu berücksichtigen, dass die Bevölkerung älter wird. Freiwilliges Engagement wird somit zukünftig mehr denn je notwendig sein, um die Betreuung und Begleitung von Menschen sicher zu stellen, die altersbedingt in sozialen Einrichtungen leben oder durch sie betreut werden. Gleichzeitig steigt aber auch die 
Zahl derjenigen Seniorinnen und Senioren, die physisch und psychisch in der Lage dazu sind, ihre gesellschaftliche Teilhabe durch freiwilliges Engagement realisieren zu können.

\section{So kann es gehen}

Über die Identifizierung unterschiedlicher Formen und Möglichkeiten freiwilligen Engagements gilt es, in den Organisationen systematische Passungen vorzunehmen und ein Freiwilligenmanagement aufzubauen. Die Öffnung der Organisationen, Einrichtungen und Dienste für freiwilliges Engagement und ihre Einbindung und Verankerung in das Gemeinwesen ist eine zentrale Anforderung. Dabei sind verschiedene Faktoren zu berücksichtigen:

- Konzept: Es ist zu klären, ob es bereits Vorstellungen zur systematischen Zusammenarbeit mit Freiwilligen gibt. Die Selbstverpflichtung aller Beteiligten, die systematische Zusammenarbeit mit Freiwilligen zu gestalten, ist für einen langfristigen Erfolg wichtig. Daneben ist die eigene Motivation für den Einsatz von Freiwilligen, die Ziele des Freiwilligenmanagements, die Einsatzmöglichkeiten und Aufgabenprofile zu klären.

- Bedarf: Nach dieser Klärung ist eine Bedarfseinschätzung vorzunehmen. Dies ist besonders wichtig, wenn Freiwillige neue, zusätzliche oder ergänzende Angebote schaffen sollen. Eine klare Abgrenzung zu den Aufgaben der hauptamtlichen Mitarbeitenden ist notwendig, um Konflikte zu vermeiden und Reibungsverluste zu verhindern. Die Bedürfnisse Hauptamtlicher hinsichtlich ihrer neuen Aufgaben im Freiwilligenmanagement sind zu berücksichtigen, durch Beratung, Supervision, Fortbildung etc.

- Aufgaben: Aus der Bedarfseinschätzung ergeben sich die Aufgabenentwicklung und die Aufgabenbeschreibung. Freiwillige dürfen dabei weder über- noch unterfordert werden. Zur Aufgabenentwicklung gehört auch, Voraussetzungen für ein partnerschaftliches Miteinander zwischen Hauptamtlichen und Freiwilligen zu schaffen, also Aufgaben, Zuständigkeiten und Verantwortung voneinander abzugrenzen und Rollen klar zu beschreiben. Die Hauptamtlichen sind unbedingt einzubeziehen.

- Rahmenbedingungen: Engagierte sind während ihrer Tätigkeit versichert. Es hat sich bewährt, Freiwillige mit einem Informationsblatt über ihre Rechte und Pflichten (z. B. Schweigepflicht) zu informieren. In einigen Fällen ist eine schriftliche Vereinbarung über die Aufgaben und den Umfang des Engagements zweckmäßig und hilfreich. Des Weiteren ist zu klären, wo beispielsweise der Arbeitsplatz für die Freiwilligen ist, ob sie einen Computer und Schlüssel benötigen, ob die Fahrtkosten erstattet werden können usw.

- Ansprechpersonen: Eine Ansprechperson koordiniert und begleitet die Freiwilligen. Sie ist für die Freiwilligen und die Hauptamtlichen bei allen Fragen zum bürgerschaftlichen Engagement da. Dieser Hauptamtliche ist mit den erforderlichen Kompetenzen auszustatten und muss für die Aufgaben ausreichende Ressourcen zur Ver- fügung haben. Dazu gehören Möglichkeiten der Weiterbildung, beispielsweise zum Ehrenamtskoordinator. (4)

- Personalgewinnung: Wenn die notwendigen Voraussetzungen geklärt sind, können Freiwillige mit Flyern, Postkarten, Faltblättern, Internetbörsen etc. geworben werden. Eine überzeugende Botschaft motiviert, zeigt die Wichtigkeit, die Anforderungen und den Nutzen des Engagements, mindert Zugangsbarrieren und benennt Ansprechpersonen. Persönliche Kontakte geben oft den Anstoß, sich zu engagieren - dabei helfen transparente Strukturen und eine offene und freundliche Atmosphäre. Die beste Werbung sind begeisterte Freiwillige - wobei der Gedanke verbesserter und übergreifender lokaler Kooperationen wesentlich sind. In diesem $\mathrm{Zu}$ sammenhang kommen engagementfördernden Infrastruktureinrichtungen im Gemeinwesen - beispielsweise Freiwilligenbörsen - eine zunehmend wichtigere Kooperationsfunktion zu.

- Vorstellungsgespräch: Im Erstgespräch mit engagementbereiten Bürgerinnen und Bürgern werden mögliche Tätigkeiten und Profile des Feldes sowie die Er-

\section{Anmerkungen}

(1) Bundesministerium für Familie, Senioren, Frauen und Jugend (Hg.): Freiwilliges Engagement in Deutschland 1999-2004. München 2005. Nachfolgend: Freiwilligensurvey 2004.

(2) Opaschowski, Horst: »Wohlstand « wird in den nächsten Jahren neu definiert. In: Tagesspiegel, 23. Juli 2006, Seite 32.

(3) Vgl. Erhard Eppler: Auslaufmodell Staat? Frankfurt am Main 2005.

(4) Vgl. auch Diakonisches Werk der EKD (Hg.): Qualifizierungsprogramm

Ehrenamtskoordinatorin/Ehrenamtskoordinator. Stuttgart 2004.

(5) Bundesnetzwerk Bürgerschaftliches Engagement (Hg.): Durch Qualifizierung zu mehr Kompetenz im Ehrenamt. Eine Initiative zur Förderung der Qualifizierung von Freiwilligen in den Mitgliedorganisationen des BBE. O. J.

(6) Vgl. z. B. Arbeitsstelle für kirchliche Dienste, Referat Ehrenamtliche Mitarbeit/Älteste (Hg.): Das kleine1x1 für das Dankeschön an Ehrenamtliche. Magdeburg o. J.

(7) Der Freiwilligensurvey 2004 weist darauf hin, dass es in den vergangenen zehn Jahren eine überdurchschnittliche Steigerung der Engagementbereitschaft bei Arbeitslosen, Rentnern sowie von ostdeutschen Frauen gegeben hat.

(8) Vgl. u. a. den Bericht des Bundestagsunterausschusses »Bürgerschaftliches Engagement « vom 16. Juni 2005, Seiten 44 bis 46 und 53 bis 54 .

(9) Vgl. EKD (Hg.): EKD Texte 84. Freiheit und Dienst. Eine Argumentationshilfe der Evangelischen Kirche in Deutschland zur Frage einer allgemeinen Dienstpflicht und zur Stärkung von Freiwilligendiensten. Berlin/Hannover, Juni 2006. 
wartungen an die Freiwilligen mit den Erwartungen, Motivationen, Bedürfnissen der Interessenten abgeglichen. Die künftige Zusammenarbeit wird in diesem Gespräch entwickelt und besprochen. Dazu gehört auch die Darstellung der Ziele der Institution und des Leitbildes.

- Einarbeitung: Kommt es zu einer Vereinbarung, bietet ein Einführungsprogramm Orientierung, im Rahmen dessen Freiwillige willkommen geheißen werden, erste Kontakte geknüpft werden und über Arbeitsabläufe, Sicherheitsbestimmungen, räumliche Gegebenheiten etc. informiert wird. Daran schließt sich die Einarbeitungszeit an, die von Hauptamtlichen oder anderen Freiwilligen begleitet werden kann.

- Begleitung, Unterstützung, Motivation und Beratung: Neben dem Austausch mit anderen sind regelmäßige Treffen in Form von Einzel- oder Gruppengesprächen Momente motivierender Begleitung. Hier ist der Raum für Anregungen, Kritik und mögliche Unzufriedenheit. Regelmäßige Treffen, bei denen Wissen vermittelt oder einfach Anregungen ausgetauscht werden, fördern die Integration und Identifikation der Freiwilligen. Zur Begleitung gehört die Gestaltung des freiwilliges Engagements von der Begrüßung bis zum Abschlussgespräch.

- Qualifizierung: Für viele Arbeitsfelder sind Methoden-, Fach- und Sozialkompetenz für Freiwillige wichtig. Für manche sind als Vorbereitung Qualifizierungen notwendig (z. B. Telefonseelsorge). Je nach Arbeitsfeld können Supervision, kollegiale Beratungen, Teambesprechungen und Austauschtreffen geeignete Orte sein. Den Freiwilligen die Teilnahme daran zu ermöglichen, ist auch ein Zeichen der Wertschätzung. (5)

- Anerkennungskultur: Die Anerkennung der Leistungen Freiwilliger ist ein zentraler Bestandteil für deren Motivation und Bindung. Es geht darum, Ihnen Ansehen und Anerkennung zu geben sowie Positives und noch ungelebte Möglichkeiten hervorzuheben. Kommunen, Gemeinden, Institutionen, Einrichtungen und Dienste sollten daher eine eigene Anerkennungskultur entwickeln. Anerkennung erfolgt häufig direkt durch die Nutzer. Die Möglichkeit sie auszudrücken hat im Prinzip jeder Mitarbeitende. Sie muss auf jeden Fall aber auch durch die Hauptamtlichen und die Leitung der Einrichtung erfolgen. Ehrungen, Zertifikate, Zeugnisse sowie Danke-Schön-Veranstaltungen (6) und vielerlei wertschätzende Signale im Alltag sind zu intensivierende Möglichkeiten.

- Monetäre Anerkennung: Freiwilliges Engagement wird grundsätzlich unentgeltlich geleistet. Auslagen für Telefonkosten, Fahrtkosten, Weiterbildungen und geringe Aufwandsentschädigungen können übernommen werden. Auch wenn Untersuchungen betonen, dass finanzielle Motive für Engagement in der Regel nicht an erster Stelle stehen (7), haben u. a. Arbeitsgelegenheiten mit Mehraufwandsentschädigung (»Ein-Euro-Jobs«) aufgrund der finanziell prekären Situation vieler Menschen (Erwerbslosigkeit, working poor) ein größeres Gewicht bekommen. (8) Kurzfristig mag es angesichts knapper Kassen und personeller Engpässe in sozialen Einrichtungen reizvoll erscheinen, qualifizierte Freiwillige in
Regelaufgaben einzubinden. Langfristig darf dies weder zum Wegfall bezahlter Arbeitsplätze noch zu Qualitätseinbußen in der Sozialen Arbeit führen.

- Evaluation: Die Evaluation ist nicht die letzte Phase, sondern integrierter Bestandteil des Freiwillligenmanagements. Sie dient dazu, Erfahrungen systematisch auszuwerten. Dabei sind die Evaluationsinstrumente mit Bedacht zu wählen. Neben dem klassischen Fragebogen hat sich das individuelle Gespräch, aber auch eine Gruppensupervision als geeignet erwiesen. Damit solch ein Mix gelingt, müssen Betroffene in den Prozess einbezogen werden. Besonders die Hauptamtlichen und die Leitungspersonen sind zu beteiligen und müssen die Arbeit mit Freiwilligen befürworten und auf diese zu koordinierende Arbeit vorbereitet sein. Dafür sind die nötigen zeitlichen, finanziellen und fachlichen Ressourcen und Kompetenzen bereitzustellen.

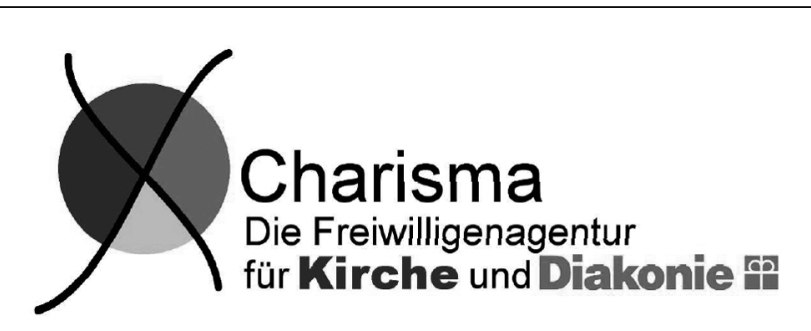

"Sie haben, was wir suchen« -
mit Ausrufe- und mit Fragezeichen

Das ist das Motto der Freiwilligenagentur »Charisma«, ein Projekt des Diakonischen Werkes Berlin - Brandenburg - schlesische Oberlausitz. Diese 2007 gegründete Freiwilligenagentur unterstützt in ihrem Zuständigkeitsbereich in erster Linie Menschen nach ihren jeweiligen individuellen Fähigkeiten, Neigungen und geografischen wie zeitlichen Möglichkeiten. Dafür unterbreitet sie in sozialen und gemeinnützigen Projekten und Einrichtungen Interessierten Engagementangebote in Kirche und Diakonie. Auch Weiterbildungsangebote für Freiwillige bietet sie an. Des weiteren sieht sie es als ihre Aufgabe an, Menschen und Einrichtungen diese Engagementfähigkeiten und Engagementfertigkeiten zugute kommen $\mathrm{zu}$ lassen. Wie so oft beim freiwilligen Engagement können dies beide Seiten als Gewinn zu betrachten, eine klassische Win-win-Situation.

Charisma, die Freiwilligenagentur für Kirche und Diakonie, Schönhauser Allee 141, 10437 Berlin, Telefon 030 44323711, Fax 03044323713 , E-Mail info@charisma-diakonie.de, Internet http://www.charisma-diakonie.de 


\section{„Warum soll ich etwas spenden? Mir schenkt auch keiner was!«}

Motivation zum Einsatz für andere und Gründe für altruistisches Handeln sind ein Dauerthema im (Religions-) Unterricht. Die Erfahrungen aus dem schulischen Alltag zeigen, dass Diskussionen zu dieser Frage kaum weiterhelfen.

Ein neuer Ansatz, den die Realschule im brandenburgischen Templin verfolgt, verlegt das Lerngeschehen in die Praxis. Ein zweitägiges diakonisch-soziales Praktikum führte 2005 alle 75 Schülerinnen und Schüler der Jahrgangsstufe 8 an Orte der Sozialen Arbeit. Das Spektrum reichte von der Be-

hindertenhilfe über ambulante, betreute und mobile Dienste für Senioren, Kindergärten, Kleiderkammern bis zur Suchtkrankenhilfe. Der Zugang der Lernenden zu ihren Einsatzfeldern gestaltete sich fast durchgehend unproblematisch. Anreiz war zugegebenermaßen auch die Aussicht, an zwei Tagen nicht in der Schulbank zu sitzen, sondern in einer anderen Umgebung etwas praktisch zu machen. Die Schule verband damit die Erwartung, dass die Lernenden in der Praxis ihre Identität erweitern. Wie die Auswertungen zeigten, gelang durch die Öffnung in eine sonst unbekannte Welt, die gänzlich anderen Lebenslagen von Menschen kennenzulernen.

Eine Schülerin berichtet von einem gelungenen Perspektivenwechsel: Menschen mit Behinderungen habe sie im Stadtbild von Templin schon oft gesehen. Nun sei sie mit einer Gruppe von Menschen mit psychischen Behinderungen selbst mit in der Stadt unterwegs gewesen und stellte fest: »Die Leute haben uns angeglotzt, dabei sind wir bloß spazieren gegangen.«

Ein Schüler, der in einem Seniorenheim eingesetzt war, lernte dort, wie wichtig Gespräche für alte Menschen sind. Ganze Lebensgeschichten wurden ihm erzählt. Der Schüler hat nicht nur zugehört, sondern ist auf die alten Menschen zugegangen. So gelang es ihm, bei einer Frau, die nicht essen wollte, die Bereitschaft zum Essen zu wecken.
Nicht nur dieser Schüler, auch die anderen fünf, die im selben Haus arbeiteten, sind überzeugt, dass Langeweile für alte Menschen in Heimen ein großes Problem ist. »Eigentlich müsste man $\mathrm{ab}$ und zu hingehen und sich mit ihnen unterhalten oder sie im Rollstuhl nach draußen schieben«, sagt einer. Der zuständige Lehrer wurde hellhörig und versuchte ein weiterführendes Projekt zu initiieren. Der Impuls wurde nicht aufgegriffen, vermutlich da er vonseiten der Schule - also von außen - und nicht vonseiten der Einrichtungen selbst kam.

Für das diesjährige Projekt soll versucht werden über die Praxisbetreuer die Einrichtungen zu ermutigen, Schülerinnen und Schüler zu weiteren Aktivitäten einzuladen. Im Idealfall bringen die jungen Menschen durch eine Identitätserweiterung aus der Praxis ein neues Selbstbild mit. »Ich kann etwas, wovon ich bisher noch nichts wusste. Ich werde von anderen Menschen gebraucht!« Der betreuende Lehrer macht deutlich: »In dieser Erfahrung sehen wir die Grundlage weiteren Engagements. Durch lebendiges Geschehen kann vermittelt werden, dass nicht nur Geben, sondern auch Nehmen von besonderer Bedeutung ist, ist man mit dem diakonisch-sozialen Lernen auf dem Weg zu Freiwilligen Engagement.«

Das Templiner Beispiel ist eines unter vielen, die als Freiwilligenprojekte mittlerweile in verschiedenen Bundesländern und Landeskirchen durchgeführt werden. Informationen im Internet:

http://www.agentur-mehrwert.de

http://www.atlas-lernorte.de

http://www.freiwilligenprojekte.de

http://ls-bw.de

http://www.szenenwechsel.de 


\section{"Zeit für Menschen«}

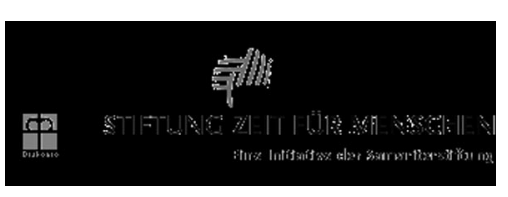

Sozialstaatliche Umbrüche sind auch in der Samariterstiftung Nürtingen spürbar. Für ein großes diakonisches Unternehmen kann diese Entwicklung nicht tatenlos hingenommen werden. Bedeutet Zeit für Zuwendung doch: Menschenwürde zu bewahren.

Deshalb haben - auf Initiative der Samariterstiftung - Bürger, Politiker und Unternehmen die Stiftung »Zeit für Menschen « gegründet. Diese Stiftung trägt nicht nur Geld, sondern vor allem Zeit und Know-how zusammen und fördert die Einbindung der Einrichtungen ins Gemeinwesen. Die Stifter wollen mit der über den finanziellen Aspekt hinausgehenden Stiftungsidee durch die Stärkung freiwilligen Engagements ein sozialpolitisches Signal zu einer erneuerten politischen Verantwortung setzen: Eine Verantwortung im Sinne einer Weiterentwicklung statt eines Abbaus sozialer Standards. Sie steht für eine Soziale Arbeit, bei der »Zeit für Menschen« zu haben kein Luxus ist oder durch Kostendruck unter die Räder gerät, sondern wo Nächstenliebe mit Hilfe freiwilligen Engagements und Bürgerverantwortung einen neuen Stellenwert bekommt.

Seit 2004 ist diese Stiftung aktiv und unterstützt die Samariterstiftung in vielfältiger Weise im Bemühen, Bürgerinnen und Bürger für soziales Engagement zu gewinnen und diese Arbeit in Form eines Stiftungsvermögens zu finanzieren. Dabei geht die Stiftung neue Wege, indem sie regionale Kooperationen beispielsweise zwischen den Einrichtungen und Unternehmen, Schulen, Kirchengemeinden und Vereinen anstößt und moderiert. Nach zwei Jahren kann eine überaus erfolgreiche Zwischenbilanz gezogen werden:

- Seit Jahren engagieren sich Auszubildende und Mitarbeitende der Firma Bosch in Reutlingen im Samariterstift Grafeneck: Sie legen in Wochenendeinsätzen Grillstellen und Sinnespfade an, restaurieren Grabsteine, feiern mit den Bewohnerinnen und Bewohnern oder begleiten Sie zu Fußballspielen.

- Das Projekt »Lebensqualität durch bürgerschaftliches Engagement« konnte in einigen Teileinrichtungen initiiert werden. Im Vordergrund steht dabei der strukturelle Auf- und Ausbau des freiwilligen Engagements.

- Die Stiftung bezuschusst generationsübergreifende Projekte und pflegt enge Kontakte mit Schulen, Ausbildungsstätten und Lehrbetrieben.

- Eine Kooperation mit der Firma Paul Hartmann AG, die auch Stifter bei »Zeit für Menschen« ist, ermöglicht im Juli 2006 einen Aktionstag auf der Landesgartenschau, indem das Unternehmen 100 Beschäftigte der Ostalb-Werkstätten zu Führungen und Austausch einlädt.

- Die unter dem Dach der Stiftung gegründete »Münsinger Alb Stiftung « unterstützt speziell einzelne Einrichtungen, zum Beispiel indem sie einen Beraterkreis von Firmen für die Werkstatt für behinderte Menschen in Münsingen initiiert.

Internet http://www.zeit-fuer-menschen.de
Berufsbegleitender

\section{Masterstudiengang}

\section{Sozialinformatik}

Erstmals in Deutschland:

Qualifizierte Hochschul-Ausbil-

dung zum IT-Experten für die

Sozialwirtschaft

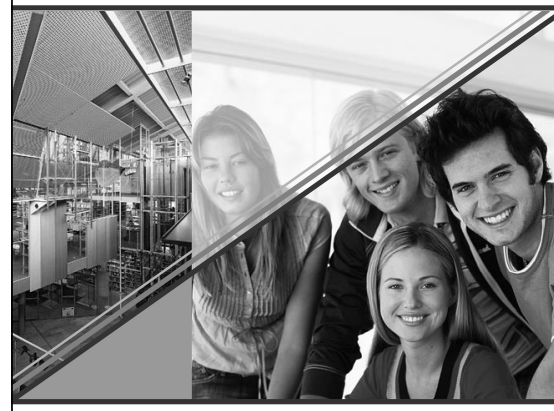

- Dauer: 4 Semester, Blockveranstaltungen + E-Learning

- Start: Oktober 2009

- Bewerbungsschluss:

31. 7. 2009

- Abschluss: Master of Arts

- Studiengangsleitung:

Prof. Helmut Kreidenweis

Gerne senden wir Ihnen eine ausführliche Studiengangsbeschreibung und weitere Informationen zu.

Kath. Universität

Eichstätt-Ingolstadt

Fakultät für Soziale Arbeit

Ostenstr. 26

85071 Eichstätt

sozialinformatik-master@ku-eichstaett.de

sozialinformatik.de/master
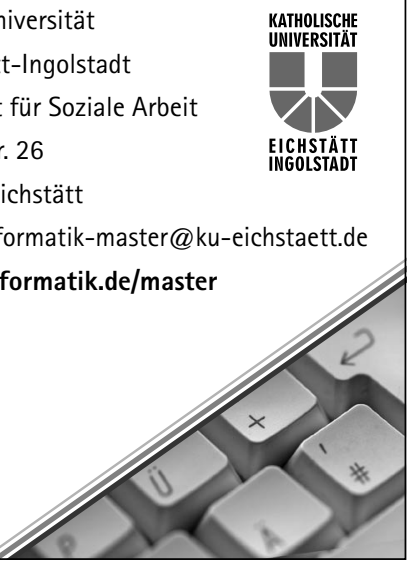